\title{
Digitale Souveränität in der Wirtschaft - Gegenstandsbereiche, Konzepte und Merkmale
}

\author{
Ernst Andreas Hartmann ${ }^{(\bowtie)}$ \\ Institut für Innovation und Technik (iit), Steinplatz 1, 10623 Berlin, Deutschland \\ hartmanneit-berlin.de
}

\begin{abstract}
Zusammenfassung. Das Konzept der digitalen Souveränität wurde auf verschiedene Gegenstände (Staaten, Organisationen, soziale Bewegungen, Individuen) angewandt und unter verschiedenen Aspekten (nationale Souveränität, Datenschutz/Datensicherheit, Wettbewerbsfähigkeit, Bürgerrechte, Arbeitsgestaltung) diskutiert. Diese Gegenstände und Aspekte werden zunächst im Überblick dargestellt. Anschließend steht die digitale Souveränität in der Wirtschaft im Fokus. Für diesen Gegenstandsbereich werden zwei Arten von Akteuren als konstitutiv angenommen: Individuen (Personen) und Organisationen (korporative Akteure, zum Beispiel Unternehmen). Unter Rückgriff auf psychologische (Hacker) und soziologische (Coleman) Handlungstheorien wird das Konstrukt der Handlung als zentrale Beschreibungsdimension der digitalen Souveränität vorgeschlagen. Speziell das Konzept der effizient-divergenten Handlungsräume nach Rainer Oesterreich, das handlungs- mit kontrolltheoretischen Aspekten verbindet, weist Potenziale auf, digitale Souveränität nicht nur theoretisch präzise zu fassen, sondern auch als Grundlage für die praktische Messung - und perspektivisch die Zertifizierung - digitaler Souveränität in Wirtschaft und Arbeitswelt dienen zu können.
\end{abstract}

Schlüsselwörter: Digitale Souveränität · Handlungstheorie · Kontrolltheorie · Organisationsgestaltung $\cdot$ Technikgestaltung $\cdot$ Arbeitsgestaltung

\section{Einleitung und Überblick}

Das Konzept der digitalen Souveränität hat durch die zunehmende Digitalisierung aller gesellschaftlichen Bereiche - Wirtschaft und Arbeit, Bildung, Politik und Verwaltung, privates und soziales Leben - eine große Aufmerksamkeit in der fachlichen und öffentlichen Diskussion gewonnen. In einer früheren Publikation des Instituts für Innovation und Technik (iit) in der VDI/VDE Innovation + Technik GmbH (Wittpahl 2017) wurden etwa folgende Themen als wesentlich für eine Auseinandersetzung mit digitaler Souveränität erachtet:

- Dimension Bürger

- Digitale Souveränität in sozialen Netzwerken und ihre Bedrohung am Beispiel der Social Bots

- Digitale Partizipation in Wirtschaft (zum Beispiel Open Innovation) und Wissenschaft (zum Beispiel Open Science/Citizen Science)

(C) Der/die Autor(en) 2021

E. A. Hartmann (Hrsg.): Digitalisierung souverän gestalten, S. 1-16, 2021.

https://doi.org/10.1007/978-3-662-62377-0_1 
- Soziodigitale Souveränität als souveräne Haltung und Kompetenz von Individuen gegenüber sozial und kulturell eingebetteten digitalen Technologien

- Dimension Unternehmen

- Digitale Souveränität als technologische Souveränität im Bereich digitaler Technologien wie etwa im Kontext Industrie 4.0, im Hinblick auf Bewertung, Nutzung und Herstellung solcher Technologien

- Datenschutz und Privatheit in der digitalen Arbeitswelt

- Dimension Staat

- Spannungsverhältnis zwischen Big Data/Data Science und traditioneller Statistik in der Beschreibung und Analyse politischer und gesellschaftlicher Realität

- Staatliches Handeln zur Erhaltung und Förderung digitaler Souveränität im Kontext der ,Sphären“ Individuum, Organisation/Unternehmen, Nationalstaat und zwischenstaatliche/internationale/transnationale Akteure

- Bildung für digitale Kompetenzen als zentrale Voraussetzung für digitale Souveränität auf allen Ebenen.

Gegenüber dieser sehr breiten Auffassung von digitaler Souveränität gibt es auch andere Konzepte, die etwa stärker auf eine staatspolitische (zum Beispiel Misterek 2017, Timmers 2019) oder staats- und völkerrechtliche (zum Beispiel Schaar 2015) Sichtweise setzen und/oder Aspekte des Datenschutzes und der Datensicherheit stärker in den Vordergrund rücken (zum Beispiel Posch 2017, aus Verbraucherschutzperspektive auch SVRV 2017). Im folgenden Kapitel sollen diese unterschiedlichen Kontexte und Konzepte digitaler Souveränität noch etwas systematischer dargestellt werden.

Für die Zwecke des hier vorliegenden Bandes - und des dahinterstehenden Projekts „Digitale Souveränität in der Wirtschaft“ - sollen Fragen der digitalen Souveränität aus der Perspektive von zwei Arten von Akteuren betrachtet werden: Individuellen Personen in ihrer Rolle als Beschäftigte in der Wirtschaft einerseits und Unternehmen andererseits.

Beide Arten von Akteuren können zurückgreifend auf Hacker $(2005,2010)$ und Coleman (1984) als Initiatoren und Träger zielgerichteter Handlungen interpretiert werden. In diesem Sinne soll digitale Souveränität in der Wirtschaft als Handlungsfähigkeit von Individuen und Unternehmen in einer zunehmend digitalisierten Welt verstanden werden. Sehr eng verbunden mit dem Konzept der Handlungsfähigkeit sind Autonomie (Hackman und Oldham 1975) und Kontrolle im psychologischen Sinne der Kontrolle über die eigenen Arbeits- und Lebensumstände (Oesterreich 1981, Luchman und González-Morales 2013). Rainer Oesterreich (1981) verband Handlungs- und Kontrolltheorie in einer Weise, die große Potenziale zur Beschreibung und Analyse digitaler Souveränität aufweist. Diese Potenziale werden im Hinblick auf die beiden Akteurtypen Individuum und Organisation erkundet und dargestellt, mit Ausblicken auf die Analyse, Zertifizierung und Gestaltung von Systemen - im Sinne einzelner technischer Systeme wie auch komplexer Anwendungssituationen - in ihren Auswirkungen auf die digitale Souveränität von Individuen und Organisationen. 


\section{Kontexte und Bedeutungshintergründe digitaler Souveränität}

In einer Überblicksarbeit beschreiben Stephane Couture und Sophie Toupin (2019) basierend auf einer Analyse sowohl wissenschaftlicher Literatur wie auch von Texten aus gesellschaftlichen und politischen Kontexten eine ganze Reihe von Bedeutungshintergründen für digitale Souveränität. Sie unterscheiden fünf Konzepte digitaler Souveränität, die sich (bis auf das zweite, siehe unten) mehr oder weniger stark abgrenzen von traditionellen Vorstellungen nationalstaatlicher Souveränität.

Das erste dieser Konzepte nennen die Autorinnen „Cyberspace-Souveränität“. Dieses Konzept bezeichnet die Vorstellung, dass das Internet selbst ein „virtuelles Territorium“ darstellt, das nicht notwendigerweise der Souveränität traditioneller Nationalstaaten untersteht - und, so meinen manche, auch nicht unterstehen sollte. Mitunter werden auch Multistakeholder-Foren wie das Internet Governance Forum (IGF) und Organisationen wie die Internet Corporation for Assigned Names and Numbers (ICANN) als Ausdrucksformen einer Souveränität der Akteure des Internets - jenseits nationalstaatlicher Souveränität - interpretiert.

Das zweite Konzept bezeichnet - komplementär oder auch in Konkurrenz zum ersten - die digitale Souveränität der Staaten und Regierungen. Diese Perspektive steht in der Tradition der in der zweiten Hälfte des 20. Jahrhunderts viel diskutierten Vorstellungen von und Forderungen nach technologischer Souveränität der Nationalstaaten, im Sinne von Verfügung über Technologien zur Förderung der eigenen Belange, wie etwa der eigenen Industrie und Innovationsfähigkeit. Sie bezieht sich auch unmittelbar auf die ursprüngliche Vorstellung von Souveränität als legitimierte und effektive Herrschaft über Territorien.

Der dritte Bedeutungshintergrund - vielleicht etwas ungewohnt aus europäischer Perspektive (die Autorinnen sind Kanadierinnen) - betrifft die indigene digitale Souveränität. Dahinter steht die Vorstellung, digitale Technologien für die Selbstbestimmung und die Selbstregierung der indigenen Völker - First Nations - zu nutzen, wobei der aktuelle Stand der Verfügung indigener Gruppen über diese Technologien als niedrig eingeschätzt wird. Einerseits im Kontrast, andererseits aber auch in Analogie zur oben besprochenen Souveränität der nationalstaatlichen Regierungen geht es hier um die digitale Souveränität der Völker und Nationen ohne Nationalstaat und ohne staatliche Souveränität.

Viertens unterscheiden die Autorinnen die digitale Souveränität sozialer Bewegungen. Hier spielen die Nutzung und eventuell auch die Entwicklung von Open Source Software ebenso eine besondere Rolle wie die eigenverantwortliche Verwendung von Verschlüsselungstechnologien. Die digitale Souveränität sozialer Bewegungen steht im Kontrast zur digitalen Souveränität und zur Macht von Regierungen und großen IT-Unternehmen.

Fünftens gibt es schließlich die persönliche digitale Souveränität, die digitale Souveränität der Individuen. In ähnlicher Weise wie bei der digitalen Souveränität der sozialen Bewegungen geht es hier weniger um eine passive Perspektive auf das 
Individuum als Objekt staatlicher Schutzmaßnahmen, etwa im Kontext der Datenschutzgesetzgebung. Vielmehr wird eine aktive Rolle des Individuums in den Vordergrund gestellt, was sich etwa in einem kompetenten Umgang mit den eigenen Daten oder auch in der gezielten Nutzung von Verschlüsselungstechnologien äußert.

Die Autorinnen identifizieren eine Reihe von Gemeinsamkeiten und Unterschieden in den Sichtweisen auf digitale Souveränität, die sie in ihrer Analyse identifiziert haben.

Erstens beobachten sie in der Entwicklung der Diskussion über die Zeit eine Tendenz weg von einer fast ausschließlichen Betrachtung der staatlichen Ebene hin zu einer stärkeren Wahrnehmung von zivilgesellschaftlichen Gruppen und Individuen.

Zweitens erkennen sie das Konzept der digitalen Souveränität als geprägt von Ideen wie Unabhängigkeit, Autonomie und Kontrolle im Sinne von „control“, also der Macht von Entitäten beziehungsweise (kollektiven) Subjekten - Staaten, Organisationen und Individuen - ihre Umwelt und ihre Lebensbedingungen zu kontrollieren, unter ihrer Kontrolle zu haben. Diese Unabhängigkeit, Autonomie und Kontrolle äußern sich in zwei Richtungen, erstens im Hinblick auf die Nutzung der Chancen der Technologien, der produktiven Aneignung der Technologien und ihrer Möglichkeiten für eigene Zwecke der jeweiligen individuellen oder kollektiven/ korporativen Akteure, zweitens im Hinblick auf den Schutz der Privatheit und der Datenhoheit aller dieser Akteure.

Drittens steht hinter den Konzepten der digitalen Souveränität oft der Anspruch, eine Gegenmacht zu sehr starken Akteuren - wie etwa den großen US-amerikanischen IT-Konzernen - aufzubauen.

Neben diesen Gemeinsamkeiten sehen die Autorinnen auch teilweise grundlegende Unterschiede zwischen den verschiedenen Konzepten digitaler Souveränität. So verwenden etwa autoritäre Staaten das Konzept staatlicher digitaler Souveränität dazu, weitreichende Formen der Machtausübung - einschließlich der Überwachung und Zensur digitaler Daten und Kommunikation - im Bereich ihrer Jurisdiktion bzw. im Bereich ihrer effektiven Handlungsfähigkeit zu realisieren und zu legitimieren. Soziale Bewegungen demgegenüber sehen ihre digitale Souveränität als Mittel der Abwehr einer solchen staatlichen Kontrolle und Herrschaftsausübung.

Vergleicht man nun die von Couture und Toupin (2019) beschriebenen fünf Kontexte der digitalen Souveränität - Cyberspace-Souveränität, Souveränität der Nationalstaaten, der Indigenen, der sozialen Bewegungen und der Individuen - mit den in dem von Volker Wittpahl (2017) herausgegebenen Band adressierten Akteuren Bürger, Unternehmen und Staat, wie oben in der Einleitung dargestellt, so fällt Folgendes auf:

- Staat und Individuen/Bürger werden in beiden Konzepten als Akteure und Träger digitaler Souveränität benannt.

- Die sozialen Bewegungen und indigenen Gruppen/Nationen fehlen in dem Band von Wittpahl (2017).

- Couture und Toupin (2019) wiederum betrachten Individuen nur als private Personen und nicht als arbeitende Menschen, Beschäftigte in Unternehmen oder anderen Organisationen. 
- Unternehmen und andere Organisationen - abgesehen von solchen, die man den sozialen Bewegungen zurechnen könnte - werden von Couture und Toupin (2019) gar nicht als Subjekte digitaler Souveränität wahrgenommen, im Gegensatz zu den Konzepten bei Wittpahl (2017). Ein möglicher Grund dafür könnte sein, dass die digitale Souveränität der Unternehmen, ihre Handlungsfähigkeit in digitalen Umwelten gar nicht als fraglich oder bedroht wahrgenommen wurde. Demgegenüber stehen Befunde, die die digitale Souveränität zumindest von kleinen und mittleren Unternehmen (KMU) als prekär, bedroht und entwicklungsbedürftig erscheinen lassen (vgl. zum Beispiel den Beitrag 2 von Annelie Pentenrieder, Anastasia Bertini und Matthias Künzel in diesem Band).

Im Folgenden soll - die Darstellung bei Wittpahl (2017) aufgreifend - die digitale Souveränität in der Wirtschaft im Vordergrund stehen. Insofern wird hier ein bedeutsamer Kontext der digitalen Souveränität zum Gegenstand gemacht, der in der breiten fachlichen und öffentlichen Diskussion eher weniger thematisiert wird (Couture, Toupin 2019). Dabei werden sowohl Individuen in ihrer Arbeitswelt wie auch Unternehmen (Organisationen) als Träger der digitalen Souveränität betrachtet.

\section{Digitale Souveränität in der Wirtschaft}

\subsection{Handlungsfähigkeit und Kontrolle von Individuen und Organisationen}

Die Gestaltung von Arbeitswelten im Sinne der arbeitenden Menschen ist eine Kerndomäne der Arbeitspsychologie (Kleinbeck und Schmidt 2010). Eine bedeutsame Denkrichtung innerhalb der Arbeitspsychologie - die „Dresdner Schule“ - stellt eine Theorie des menschlichen Handelns - die Handlungsregulationstheorie - in das Zentrum der Analyse und Gestaltung menschlicher Arbeit (Hacker 2005, 2010, Hacker und Richter 1990).

Neben individuellen Menschen können (beispielsweise nach dem grundlegenden theoretischen Ansatz von Coleman 1986) auch Organisationen wie etwa Unternehmen als korporative Akteure und somit auch als handelnde Subjekte betrachtet werden. Digitale Souveränität in der Wirtschaft wäre also in erster Näherung als Erhalt und möglichst Förderung der Handlungsfähigkeit von Individuen und Organisationen in wirtschaftlichen Kontexten zu verstehen.

Bereits oben in der Diskussion des Beitrags von Couture und Toupin (2019) wurde deutlich, dass Konzepte wie Unabhängigkeit, Autonomie und Kontrolle - im Sinne einer Kontrolle der individuellen oder korporativen Subjekte über ihre Umweltbedingungen - sehr nah mit der digitalen Souveränität verbunden sind, sie näher beschreiben können. In der Arbeitspsychologie gilt dies vor allem für das Konstrukt der Kontrolle als wesentliches, gerade auch für psychische und physische Gesundheit überragend bedeutsames Merkmal von Arbeitsbedingungen (vgl. grundlegend zum Beispiel Karasek 1989, für eine Überblicksdarstellung in deutscher Sprache Flammer, 
Nakamura 2002, für einen Überblick über die empirischen Befunde de Jonge und Kompier 1997 sowie Luchman und González-Morales 2013).

Handlungs- und kontrolltheoretische Aspekte verknüpft Rainer Oesterreich (1981) in seinem Konzept des Handlungsraums und der Effizienz-Divergenz in Handlungsräumen. Im folgenden Abschnitt wird dieses Konzept etwas eingehender dargestellt, einschließlich der recht abstrakt-formalen Definitionen, die Oesterreich zur Beschreibung von Effizienz und Divergenz verwendet. Diese formalen, expliziten, mathematischen Beschreibungen werden auch deshalb dargestellt, weil sie für eine eventuelle spätere Verwendung des Effizienz/Divergenz-Konzepts im Kontext der Zertifizierung von digitalen Anwendungen und Produkten im Werkzeugmaschinenbau nützlich sein können, zur Operationalisierung der Bewertungskriterien.

Vor dieser formalen Darstellung sollen die Grundgedanken des Konzepts kurz qualitativ umrissen werden:

- Die Umwelten von Menschen und Organisationen lassen sich verstehen als Handlungsräume, in denen die handelnden Subjekte in einer bestimmten Situation Handlungsmöglichkeiten vorfinden. Von diesen Handlungsmöglichkeiten wählen sie eine aus, was sie zu einer neuen Situation führt, in der wiederum mehrere Handlungsmöglichkeiten bestehen (und so weiter, bis hin $\mathrm{zu}$ sehr komplexen Handlungsräumen).

- Die Effizienz einer solchen Situation bzw. eines Handlungsraums hängt nun davon $\mathrm{ab}$, inwieweit sich von einer Situation aus durch die Auswahl geeigneter Handlungen möglichst sicher (mit hohen Wahrscheinlichkeiten) bestimmte Folgesituationen erreichen lassen.

- Die Divergenz hängt demgegenüber davon ab, wie viele unterschiedliche Folgesituationen von einer bestimmten Situation mit den dort verfügbaren Handlungen erreichbar sind.

- Effizienz-Divergenz kombiniert als Maß beide Aspekte

\subsection{Handlungsraum, Effizienz und Divergenz: Formale Definitionen}

Die oben grob qualitativ umrissene Beschreibung der Konzepte Handlungsraum Effizienz, Divergenz und Effizienz-Divergenz werden im Folgenden präziser und formal beschrieben.

Ein Handlungsraum kann zunächst verstanden werden als ein gerichteter Graph mit Situationen/Zuständen als Knoten und Handlungen als gerichteten - von der Gegenwart in die Zukunft, von einem Zeitpunkt zum nächsten, von tn $\mathrm{zu}$ tn+1 Kanten. Da jede Situation wiederum als Ergebnis einer vorangegangenen Handlung interpretiert werden kann, heißen die Knoten beziehungsweise die Situationen in Oesterreichs Terminologie Konsequenzen, formal bezeichnet mit dem Symbol K.

Abb. 1 zeigt einige Beispiele für einfache Handlungssequenzen. Für jede Ausgangskonsequenz $\mathrm{K}_{\text {tn }}$ stehen mehrere Handlungen $\mathrm{H}_{1} \ldots \mathrm{H}_{\mathrm{n}}$ zur Verfügung. Jede dieser Handlungen führt entweder sicher zu einer bestimmten Konsequenz $\mathrm{K}_{\mathrm{tn}+1} \mathrm{zum}$ folgenden Zeitpunkt, oder sie führt mit Wahrscheinlichkeiten von jeweils kleiner als $1 \mathrm{zu}$ mehreren Konsequenz $\mathrm{K}_{\mathrm{tn}+1}$ zum nächsten Zeitpunkt. Jede der Konsequenzen $\mathrm{K}_{\mathrm{tn}+1}$ zum zweiten Zeitpunkt kann nun wieder als Ausgangskonsequenz verstanden 
werden, für die mehrere Handlungen zur Verfügung stehen, die wiederum zu neuen Konsequenzen $\mathrm{K}_{\mathrm{t}+2}$ führen, und so weiter. Komplexe Handlungsräume lassen sich so aus den in Abb. 1 dargestellten einfachen Elementen als Bausteinen zusammensetzen.

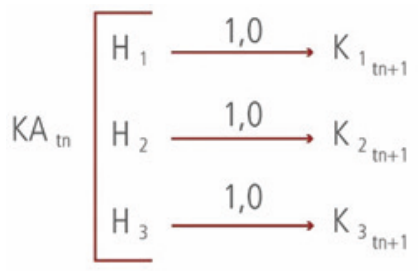

$$
E D=1,0
$$

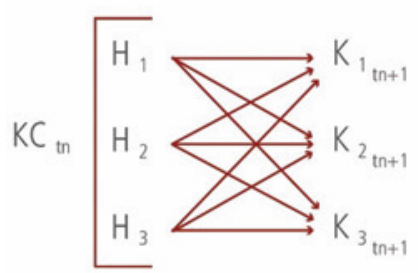

$$
\begin{gathered}
\mathrm{p}(\longrightarrow)=0,3^{-} \\
\mathrm{ED}=\mathbf{0 , 0}
\end{gathered}
$$

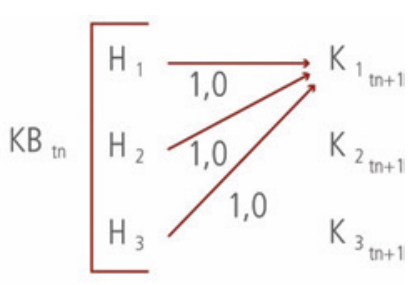

$$
E D=0,0
$$

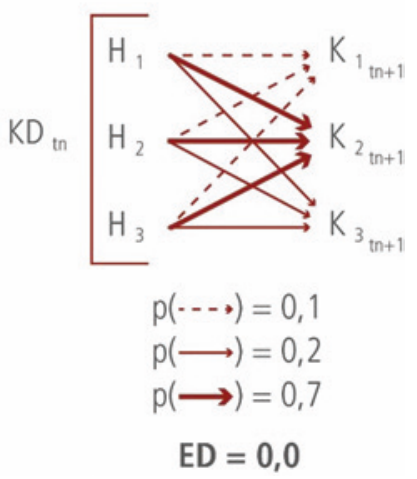

Abb. 1. Einige Beispiele für Effizienz-Divergenz (ED) bei einfachen Handlungssequenzen

Die Effizienz eines Handlungsraums wird nun dadurch bestimmt, wie hoch die Übergangswahrscheinlichkeiten der Handlungen sind, die von den Konsequenzen $\mathrm{K}_{\mathrm{tn}} \mathrm{zu}$ den Konsequenzen $\mathrm{K}_{\mathrm{tn}+1}$ führen. Idealerweise führt jede Handlung sicher mit $\mathrm{p}=1,0-\mathrm{zu}$ einer bestimmten Konsequenz $\mathrm{K}_{\mathrm{tn}+1}$, wie in Abb. 1 links oben für die Konsequenz $\mathrm{KA}_{\text {tn }}$ dargestellt.

Oesterreich (1983) schlägt als formale Beschreibung der Effizienz folgenden Ausdruck vor $^{1}$ :

$$
\mathrm{E}\left(\mathrm{K}_{\mathrm{tn}}\right)=\frac{\mathrm{N}\left(\mathrm{K}_{\mathrm{tn}+1}\right) \cdot \operatorname{maxp}\left(\mathrm{H}_{1} \ldots \mathrm{H}_{\mathrm{n}}\right)-1}{\mathrm{~N}\left(\mathrm{~K}_{\mathrm{tn}+1}\right)-1}
$$

1 Nach dieser Formel hängt die Effizienz nur von der maximalen Wirkwahrscheinlichkeit einer Handlung der Ausgangskonsequenz ab, nicht - was die Situation differenzierter abbilden würde - von der Verteilung der Übergangswahrscheinlichkeiten aller Handlungen. Diese Beschränkung kann überwunden werden, wenn man in Anlehnung an Osterloh (1983) Effizienz, Divergenz und Effizienz-Divergenz als Entropien im informationstheoretischen Sinn modelliert. 
Dabei sind $\mathrm{N}\left(\mathrm{K}_{\mathrm{tn}+1}\right)$ die Anzahl der Konsequenzen zum Zeitpunkt tn+1 und $\operatorname{maxp}\left(\mathrm{H}_{1} \ldots \mathrm{H}_{\mathrm{n}}\right)$ die maximale Wirkwahrscheinlichkeit der zum Zeitpunkt tn verfügbaren Handlungen, also die maximale Wahrscheinlichkeit, mit der eine Handlung des früheren Zeitpunkts zu einer bestimmten Konsequenz des späteren Zeitpunkts führt.

Nun beschreibt eine hohe Effizienz alleine noch nicht eine für den handelnden Menschen befriedigende Situation. Dies illustriert die in Abb. 1 rechts oben für die Konsequenz $\mathrm{KB}_{\mathrm{tn}}$ dargestellte Situation. Hier herrscht maximale Effizienz, alle Handlungen der Ausgangskonsequenz $\mathrm{K}_{\mathrm{tn}}$ führen sicher mit der Wahrscheinlichkeit $\mathrm{p}=1,0$ zu einer Folgekonsequenz $\mathrm{K}_{\mathrm{tn}+1}$; es handelt sich aber immer um dieselbe Konsequenz. Damit tritt unabhängig von der Handlungsentscheidung des Menschen zum Zeitpunkt tn immer derselbe Zustand in tn+1 ein. In einer solchen Situation kann man nicht sinnvoll von einer Kontrolle des Menschen sprechen.

Die Effizienz bedarf somit, um Kontrolle sinnvoll abbilden zu können, einer Ergänzung. Oesterreich schlägt für dieses komplementäre Konzept die Divergenz vor, verstanden als die Vielfalt von mit Handlungen des früheren Zeitpunkts erreichbaren Konsequenzen des späteren Zeitpunkts, die er wie folgt formal darstellt:

$$
\mathrm{D}\left(\mathrm{K}_{\mathrm{tn}}\right)=\frac{\mathrm{N}\left(\mathrm{K}_{\mathrm{tn}+1, \mathrm{p}>0}\right)-1}{\mathrm{~N}\left(\mathrm{~K}_{\mathrm{tn}+1}\right)-1}
$$

Dabei ist $N\left(\mathrm{~K}_{\mathrm{tn}+1, \mathrm{p}>0}\right)$ die Anzahl der zum Zeitpunkt $\mathrm{tn}+1$ mit einer Wahrscheinlichkeit größer Null erreichbaren Konsequenzen. Die Divergenz ist konzeptionell sehr nahe an den arbeitspsychologischen Konzepten der Freiheitsgrade und des Handlungsspielraums (Hacker 1987, 2005; Osterloh 1983).

Auch die Divergenz alleine kann die Kontrolle beziehungsweise Kontrollierbarkeit eines Handlungsfelds aus Sicht des handelnden Menschen nicht adäquat abbilden. Dies illustrieren die in Abb. 1 unten für die Konsequenzen $\mathrm{KC}_{\mathrm{tn}}$ und $\mathrm{KD}_{\mathrm{tn}}$ dargestellten Situationen: Hier herrscht zwar jeweils vollständige Divergenz, weil alle Konsequenzen des Zeitpunkts $t n+1$ durch Handlungen des Zeitpunkts tn erreicht werden können. Das Eintreten einer jeweils bestimmten Konsequenz zum Zeitpunkt tn+1 ist allerdings völlig unabhängig von den Handlungen und den zu ihnen führenden Entscheidungen zum Zeitpunkt tn. Die Ausgangskonsequenzen $\mathrm{KC}_{\mathrm{tn}}$ und $\mathrm{KD}_{\mathrm{tn}}$ unterscheiden sich nur dahingehend, ob die Konsequenzen des Zeitpunkts tn+1 mit gleichen oder unterschiedlichen Wahrscheinlichkeiten erreicht werden; in beiden Fällen geschieht dies aber völlig unabhängig von den zum Zeitpunkt tn gewählten Handlungen.

Aus diesen Überlegungen wird deutlich, dass für eine (formale) Beschreibung der Kontrolle beziehungsweise der Kontrollierbarkeit eines Handlungsraums ein Konzept benötigt wird, das Effizienz und Divergenz berücksichtigt. Ein solches Konzept schlägt Oesterreich als Effizienz-Divergenz (ED) vor, das er wie folgt formal beschreibt:

$$
\mathrm{ED}\left(\mathrm{K}_{\mathrm{tn}}\right)=\frac{\left(\sum_{\mathrm{i}=1}^{\mathrm{N}\left(\mathrm{K}_{\mathrm{tn}+1}\right)} \operatorname{maxp}\left(\mathrm{K}_{\mathrm{i}_{\mathrm{tn}+1}}\right)\right)-1}{\mathrm{~N}\left(\mathrm{~K}_{\mathrm{tn}+1}\right)-1}
$$


Dabei ist $\operatorname{maxp}\left(\mathrm{K}_{\mathrm{i}_{\mathrm{tn}+1}}\right)$ die maximale Wirkwahrscheinlichkeit, die zur Konsequenz $\mathrm{K}_{\mathrm{i}_{\mathrm{tn}+1}}$ führt. Die Effizienz-Divergenz wird also umso größer, je mehr Konsequenzen des Zeitpunkts tn+1 mit jeweils möglichst hohen Wirkwahrscheinlichkeiten erreicht werden können. Dies ist optimal im Fall der in Abb. 1 links oben für die Konsequenz $\mathrm{KA}_{\text {tn }}$ dargestellte Situation gegeben: Es sind alle Konsequenzen des Zeitpunkts $\mathrm{tn}+1$ erreichbar (Divergenz), und zwar jeweils mit maximaler Wahrscheinlichkeit - also sicher - durch jeweils eine - und nur eine - bestimmte Handlung (Effizienz). Die Effizienz-Divergenz ist somit ein geeignetes Maß für die Kontrolle in einem Handlungsfeld beziehungsweise für die Kontrollierbarkeit des Handlungsfelds.

\subsection{Soziodigitale Souveränität von Individuen und Organisationen: Möglichkeiten der Gestaltung}

Das Konzept der Effizienz-Divergenz wurde zur Konstruktion des Arbeitsanalyse- und Bewertungsverfahrens VERA (Verfahren zur Ermittlung von Regulationserfordernissen in der Arbeitstätigkeit) verwendet (Oesterreich 1984). Eine Anwendung zur Gestaltung soziotechnischer Systeme (Trist und Bamforth 1951, Ulrich 2013) im Allgemeinen oder zur Analyse digitaler Souveränität im Besonderen wurde nach Kenntnis des Autors bislang nicht untersucht. Da sich dieses Konzept, wie oben dargestellt, jedoch sehr gut eignet, um das als Kernaspekt der digitalen Souveränität diskutierte Konstrukt der Kontrolle recht explizit und präzise zu beschreiben, soll genau dies im Folgenden untersucht und dargestellt werden: Die Potenziale der Effizienz-Divergenz zur Beschreibung und Gestaltung der digitalen Souveränität sowohl von Beschäftigten wie von Unternehmen.

Im Folgenden wird zunächst versucht, aus der Perspektive der Individuen beziehungsweise der Beschäftigten wesentliche Aspekte ihrer digitalen Souveränität systematisch darzustellen.

Tab. 1 zeigt - im Sinne einer hypothetischen Zuordnung - Aspekte der soziodigitalen (vgl. auch Stubbe 2017) Souveränität in Relation zu den Aspekten der Kontrolle - Effizienz und Divergenz. Von soziodigitaler Souveränität wird hier gesprochen, weil die Interaktion des Menschen mit den digitalen Systemen eingebettet ist in eine soziale Umwelt. Im Kontext der Arbeitswelt ist es üblich, diese Umwelt als soziotechnisches System, bestehend aus den Teilsystemen Mensch, Technik und Organisation, zu verstehen (Trist, Bamforth 1951, Ulich 2013, speziell mit Bezug zu KI-Anwendungen Mueller et al. 2019). Dabei ist grundsätzlich davon auszugehen, dass die Gestaltung der Arbeitsorganisation den größten Einfluss auf die menschliche Arbeit hat, hinsichtlich sowohl Produktivität und anderen ökonomischen Kennwerten einerseits als auch Arbeitsqualität im arbeitspsychologischen Sinne andererseits. Dies liegt daran, dass die durch die Organisationsgestaltung definierte Verteilung und Kombination von Aufgaben den Kern der Arbeit, die Arbeitsinhalte, bestimmt. Die Auswirkung der Gestaltung des technischen Teils wird immer in diesem organisationalen Kontext zu bewerten sein (Hacker 1987).

Die Elemente der soziodigitalen Souveränität auf der Ebene des Individuums sind in Tab. 1 in den Spalten nach den Teilsystemen des soziotechnischen Systems Mensch, Technik, Organisation - angeordnet, in den Zeilen nach den Aspekten der Kontrolle - Effizienz und Divergenz. 
Tab. 1. Elemente der soziodigitalen Souveränität auf der Ebene des Individuums

\begin{tabular}{l|l|l|l}
\hline \multicolumn{1}{c|}{$\begin{array}{c}\text { Aspekt der } \\
\text { Kontrolle }\end{array}$} & \multicolumn{1}{|c|}{ Mensch } & \multicolumn{1}{c}{ Technik } & \multicolumn{1}{c}{ Organisation } \\
\hline $\begin{array}{l}\text { Übergeordnet/ } \\
\text { Voraussetzung }\end{array}$ & $\begin{array}{l}\text { Digitales Grundwissen/ } \\
\text { Digital Literacy }\end{array}$ & $\begin{array}{l}\text { Transparenz/Erklär- } \\
\text { barkeit }\end{array}$ & $\begin{array}{l}\text { Transparenz über Auf- } \\
\text { gaben und Entscheidungs- } \\
\text { befugnis }\end{array}$ \\
\hline Effizienz & $\begin{array}{l}\text { Aufgabenbezogenes } \\
\text { (digitales) Spezial- } \\
\text { wissen }\end{array}$ & $\begin{array}{l}\text { Technische Zuver- } \\
\text { lässigkeit, Robustheit, } \\
\text { Resilienz }\end{array}$ & $\begin{array}{l}\text { Aufgabenteilung und } \\
\text {-kombination, Soziale } \\
\text { Unterstützung }\end{array}$ \\
\hline Divergenz & $\begin{array}{l}\text { Interdisziplinäres } \\
\text { (digitales) Spezial- } \\
\text { wissen, Kompetenzen } \\
\text { als Selbstorganisations- } \\
\text { dispositionen. }\end{array}$ & $\begin{array}{l}\text { Eingriffsmöglich- } \\
\text { keiten in das System } \\
\text { auf wählbaren } \\
\text { Regulationsebenen }\end{array}$ & $\begin{array}{l}\text { Entscheidungs-, } \\
\text { Tätigkeits-, Handlungs- } \\
\text { spielräume }\end{array}$ \\
\hline
\end{tabular}

Für alle drei Teilsysteme gibt es zunächst allgemeine Voraussetzungen, die mit der Transparenz des digitalen Handlungsfelds aus der Sicht des Menschen zu tun haben. Entscheidend für das Kontrollerleben und die vom Menschen tatsächlich ausgeübte Kontrolle ist ja nicht die objektive Kontrolle, die das Handlungsfeld grundsätzlich gemäß seiner Merkmale ermöglicht, sondern die subjektive Kontrolle, die vom Menschen wahrgenommenen Kontrollmöglichkeiten. Eine in diesem Sinne elementare Voraussetzung im Bereich des Teilsystems Mensch ist eine digitale Grundbildung, eine elementare Digital Literacy (vbw 2018). Seitens des Teilsystems Organisation ist es eine grundsätzliche Transparenz über die dem Individuum zugewiesenen Aufgaben und über seine Entscheidungsbefugnis.

Die Transparenz im Bereich des technischen Teilsystems ist aktuell Gegenstand intensiver Diskussion, sofern es sich um Systeme mit Künstlicher Intelligenz (KI) handelt (zum Beispiel EPFL IRGC 2018, Zweig 2019); teilweise wird sogar bestritten, dass Transparenz von KI-Systemen möglich oder auch wünschenswert ist (Ananny und Crawford 2018). Im Einklang mit der Mehrheit der Autorinnen und Autoren soll hier allerdings die Auffassung vertreten werden, dass Transparenz im Kontext von KI-Systemen eine herausfordernde, aber prinzipiell lösbare Aufgabe ist. Dies gilt unter der Bedingung, dass - insbesondere bei komplexen, dynamischen KI-Systemen (maschinelles Lernen, neuronale Netze) - der Anspruch nicht darin besteht, transparent zu machen, wie die Algorithmen im Detail , wirklich funktionieren; dies dürfte in vielen Fällen objektiv nicht möglich sein. Demgegenüber besteht eine sinnvolle Forderung darin, dass KI-Systeme erklärbar sein sollen (Englisch: Explainable AI, XAI; Mueller et al. 2019; vgl. auch die Beiträge 4 von Florian Eiling und Marco Huber sowie 11 von Roland Vogt in diesem Band). Das bedeutet, dass das System ,hinreichend gute' Erklärungen für sein Verhalten geben kann. Zu diesem Zweck kann zum Beispiel das Verhalten eines neuronalen Netzes über einen - für den Menschen nachvollziehbaren - Entscheidungsbaum angenähert werden. Dabei muss immer bedacht werden, dass es sich bei diesen Erklärungen um Approximationen, nicht um Darstellungen der - objektiv kaum nachvollziehbaren tatsächlichen Arbeitsweise des (lernenden) Algorithmus handelt. Für die praktische 
Gestaltung erklärbarer XAI-Systeme stehen mittlerweile viele Modelle und Werkzeuge zur Verfügung (zum Beispiel Nushi et al. 2018, Mueller et al. 2019, Wang et al. 2019).

Speziell für den Aspekt der Effizienz ist seitens des Teilsystems Mensch ein hinreichendes Fachwissen erforderlich, um sich in digitalen Handlungsfeldern sicher bewegen zu können. Dies umfasst sowohl einschlägiges Domänenwissen sowie - je nach Art der eingesetzten digitalen Systeme und je nach Aufgabenzuschnitt (siehe unten) - auch digitales Spezialwissen (vgl. hierzu auch den Beitrag 2 von Thorsten Reckelkamm und Jochen Deuse in diesem Band). Seitens des Teilsystems Organisation wird durch die grundsätzliche Aufgabenteilung und -kombination bestimmt, inwieweit die jeweiligen Arbeitsaufgaben überhaupt von Menschen auf hohem und nachhaltigem Leistungsniveau bearbeitet werden können (vgl. das Konzept der vollständigen Tätigkeiten, Hacker 1987, 2005, 2010, Hacker und Richter 1990). Auch die soziale Unterstützung von Kollegen und Vorgesetzten, die die Effizienz positiv beeinflussen kann, hängt ganz wesentlich von betrieblichen Organisationskonzepten ab.

Für das technische Teilsystem sind unter dem Aspekt der Effizienz Merkmale wie technische Zuverlässigkeit, Robustheit und Resilienz der technischen Systeme. beispielsweise der KI-Systeme beziehungsweise Algorithmen, wesentlich (vgl. dazu auch den Beitrag 11 von Roland Vogt in diesem Band). Fragen der Qualität von Algorithmen sind anspruchsvoll (Zweig 2018, 2019), was teilweise noch dadurch erschwert wird, dass sich bestimmte relevante Qualitätsmaßstäbe untereinander logisch-mathematisch widersprechen (Zweig und Krafft 2018). Für die Analyse und Behebung von Fehlern beziehungsweise Fehlfunktionen in KI-Systemen wurden Methoden vorgeschlagen, die die Ressourcen von Menschen und technischen Systemen gleichermaßen nutzen (Nushi et al. 2018).

Bezüglich der Divergenz ist ein wesentliches Merkmal des Teilsystems Mensch das Vorhandensein kognitiver Ressourcen, die nicht nur die Bewältigung definierter, immer gleicher Anforderungen erlauben, sondern auch in neuen, ungewohnten, dynamischen Situationen Handlungsfähigkeit ermöglichen. Neben interdisziplinärem Wissen sind dies insbesondere Kompetenzen im von John Erpenbeck definierten Sinn als Selbstorganisationsdispositionen (Erpenbeck et al. 2017). Hinsichtlich des organisationalen Teilsystems sind Freiheitsgrade und Handlungsspielräume ganz zentrale Voraussetzungen von Divergenz (Osterloh 1983, Hacker 2005).

Für das Teilsystem Technik lassen sich eine ganze Reihe von Gestaltungsprinzipien benennen, die Divergenz fördern. Besonders soll das Ecological Interface Design (EID) hervorgehoben werden (Vicente und Rasmussen 1992, Lüdtke 2015). Dieser Gestaltungsansatz beruht auf der Vorstellung hierarchisch aufgebauter Handlungskontrolle bzw. -regulation (Hacker 2005; Rasmussen 1983). Auf den obersten dieser Regulationsebenen menschlicher Handlungen werden komplexe, zielgerichtete Handlungspläne - gegebenenfalls je nach Situation völlig neu - entwickelt (knowledge based behaviour bei Rasmussen, intellektuelle Regulationsebene bei Hacker), auf den untersten Ebenen (skill based behaviour beziehungsweise sensomotorische Regulationsebene) werden einfache sensorische, kognitive oder motorische Routinen quasi , automatisch“ abgearbeitet. Zwischen diesen Ebenen gibt es eine mittlere, die an Handlungsschemata orientiert ist (rule based behaviour bzw. 
perzeptiv-begriffliche Regulationsebene). Der EID-Ansatz läuft nun darauf hinaus, komplexe Mensch-Maschine-Schnittstellen so zu gestalten, dass die Nutzenden möglichst zu jeder Zeit die Möglichkeit haben, wahlweise auf jeder dieser Handlungsregulationsebenen in das System einzugreifen. Dies kann als ein sehr ausdifferenzierter Sonderfall eines ,On-the loop-Designs“ (EPFL IRGC 2018) verstanden werden - im Unterschied zu off-the-loop (Nutzer kann gar nicht in Systemprozesse eingreifen) und in-the-loop (Nutzer ist ständig in die Systemsteuerung eingebunden).

Ein weiteres Beispiel ist die Gestaltung von Interaktionen zwischen Menschen und Algorithmen in einer Weise, die es den Menschen erlaubt, kognitive Verzerrungen zu überwinden, wie etwa den confirmation bias, also die Neigung, solche Informationen $\mathrm{zu}$ suchen und hoch $\mathrm{zu}$ bewerten, die $\mathrm{zu}$ bereits gefassten (Vor-) Urteilen passen (Wang et al. 2019). Durch solche Mensch-Technik-Interaktionen entstehen neue Möglichleiten des menschlichen Handelns, sie wirken somit divergenzfördernd.

Tab. 2 zeigt Elemente der soziodigitalen Souveränität auf der Ebene der Organisation. Die Zeilen der Tabelle entsprechen wie oben im Fall der Individuen den beiden Aspekten der Kontrolle - Effizienz und Divergenz. In den Spalten finden sich in Anlehnung an die Dimensionen des iit-Innovationsfähigkeitsindikators ${ }^{2}$ die drei Kapitalarten Human-, Struktur- und Beziehungskapital.

Human- und Strukturkapital spiegeln die oben für die Individuen in den Kategorien Mensch und Organisation abgebildeten Sachverhalte aus der Perspektive des Unternehmens beziehungsweise der Organisation.

Humankapital bezeichnet das für die Organisation verfügbare Wissen der Beschäftigten. Im Hinblick auf die Effizienz ist dabei eher die Tiefe des (digitalen)

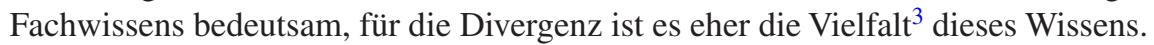

Tab. 2 Elemente der soziodigitalen Souveränität auf der Ebene der Organisation

\begin{tabular}{l|l|l|l}
\hline Aspekt der Kontrolle & \multicolumn{1}{|c|}{ Humankapital } & \multicolumn{1}{|c}{ Strukturkapital } & \multicolumn{1}{|c}{ Beziehungskapital } \\
\hline Effizienz & $\begin{array}{l}\text { Tiefe des (digitalen) } \\
\text { Fachwissens }\end{array}$ & Single-loop learning & $\begin{array}{l}\text { Verlässlichkeit der } \\
\text { (digitalen) Dienst- } \\
\text { leistungsbeziehungen }\end{array}$ \\
\hline Divergenz & $\begin{array}{l}\text { Vielfalt des (digitalen) } \\
\text { Fachwissens }\end{array}$ & Double-loop learning & $\begin{array}{l}\text { Handlungsspiel- } \\
\text { räume innerhalb der } \\
\text { (digitalen) Dienst- } \\
\text { leistungsbeziehungen }\end{array}$ \\
\hline
\end{tabular}

2 www.iit-berlin.de/de/indikator. Auf das dort zusätzlich angesprochene Komplexitätskapital wird hier aus Gründen der Darstellbarkeit verzichtet.

3 Dieser Aspekt der Vielfalt wird im iit-Innovationsfähigkeitsindikator über das Komplexitätskapital abgebildet. 
Das Strukturkapital beschreibt Potenziale der internen Strukturen der Organisation, also letztlich ihrer organisatorischen Ausgestaltung, insbesondere unter den Aspekten der Lern- und Innovationspotenziale. Dabei kommen die bekannten Modelle des organisationalen Lernens zum Tragen (Argyris und Schön 1996). Effizienz wird aufgebaut durch single-loop learning, das fortlaufende Verbessern etablierter Prozesse. Divergenz entsteht durch double-loop learning, das Entwickeln neuer organisationaler Prozesse.

Beziehungskapital schließlich betrifft die Beziehungen des Unternehmens zu externen Akteuren. Im Kontext der digitalen Souveränität sind hier besonders IT-Dienstleister von Interesse, etwa Anbieter von Cloud- und Plattformdiensten. Für die Effizienz der Organisation ist dabei die Verlässlichkeit des Anbieters wesentlich, hinsichtlich Datenschutz und -sicherheit sowie Verfügbarkeit der Daten. Die Divergenz hängt davon $\mathrm{ab}$, inwieweit der Dienstleistungsvertrag unterschiedliche Handlungsweisen zulässt, wie etwa die Speicherung von Daten an wählbaren Orten.

Denise Joecks-Laß und Karsten Hadwich beschreiben im Beitrag 6 in diesem Band unter dem Aspekt der Kundenentwicklung viele Konzepte und Methoden, die die Ausgestaltung der Dienstleistungsbeziehungen im Sinne digitaler Souveränität der Kunden unterstützen können.

\section{Zertifizierung und Gestaltung digitaler Handlungsfelder mit hoher Kontrollierbarkeit}

Die oben dargestellte Sichtweise auf digitale Souveränität unter dem Aspekt der Kontrolle - mit den Facetten Effizienz und Divergenz - erlaubt es, eine ganze Reihe von Fragestellungen, die die digitale Souveränität von Individuen und Organisationen betreffen, systematisch herzuleiten.

Für die Zukunft sollte geprüft werden, ob sich das Konzept der Kontrolle im Oesterreich'schen Sinne - vielleicht gerade auch wegen seines relativ hohen Grades an Formalisierung - dazu eignet, im Rahmen von Zertifizierungsprozessen im Kontext der digitalen Souveränität verwendet zu werden (vgl. auch den Beitrag 11 von Roland Vogt in diesem Band).

Zunächst könnten eine Operationalisierung und ein Messkonzept für Kontrolle mit den Aspekten Effizienz und Divergenz für Anwendungen digitaler Systeme entwickelt werden. Das hätte den Vorteil, dass für konkrete Anwendungsfälle der gesamte systemische Kontext digitaler Souveränität, wie oben in den Tab. 1 und 2 dargestellt, berücksichtigt werden kann. Eine solche Zertifizierung wäre interessant etwa für innerbetriebliche Regelungen zur Einführung digitaler Technologien und Prozesse.

In einem zweiten Schritt könnte geprüft werden, inwieweit dieses Messkonzept übertragbar ist auf digitale Produkte und Dienstleistungen. Eine solche Zertifizierung wäre interessant für Anbieter digitaler Produkte und Dienstleistungen als Marketing-Instrument und für deren Kunden als Orientierungshilfe bei Kauf- bzw. Kooperationsentscheidungen. 


\section{Literatur}

Ananny, M., Crawford, K.: Seeing without knowing: limitations of the transparency ideal and its application to algorithmic accountability. New Media Soc. 20(3), 973-989 (2018)

Argyris, Ch., Schön, D.A.: Organizational Learning II. Addison-Wesley, Boston/Mass (1996)

Coleman, J.S.: Social theory, social research, and a theory of action. Am. J. Sociol. 91(6), 1309-1335 (1986)

Couture, S., Toupin, S.: What does the notion of 'sovereignty' mean when referring to the digital? New Media Soc. 21(10), 2305-2322 (2019)

de Jonge, J., Kompier, M.A.J.: A critical examination of the demand-control-support model from a work psychological perspective. Int. J. Stress Manag. 4(4), 235-258 (1997)

IEPFL IRGC: The Governance of Decision-Making Algorithms. EPFL International Risk Governance Center, Lausanne (2018)

Erpenbeck, J., v. Rosenstiel, L., Grote, S., Sauter, W. (Hrsg.): Handbuch Kompetenzmessung, 3. überarbeteite und erweiterte Auflage. Schäffer-Poeschel, Stuttgart (2017)

Flammer, A., Nakamura, Y.: An den Grenzen der Kontrolle. In: Jerusalem, M. Hopf, D. (Hrsg): Selbstwirksamkeit und Motivationsprozesse in Bildungsinstitutionen (Zeitschrift für Pädagogik, 44. Beiheft). Beltz, Weinheim und Basel (2002)

Hacker, W.: Software-Ergonomie; Gestalten Rechnergestützter Geistiger Arbeit?! In: Schönpflug, W., Wittstock, M. (Hrsg.) Software-Ergonomie '87 Nützen Informationssysteme dem Benutzer? Berichte des German Chapter of the ACM. Vieweg+Teubner, Wiesbaden (1987)

Hacker, W.: Allgemeine Arbeitspsychologie, 2., vollständig überarbeitete und ergänzte Aufl. Huber, Bern (2005)

Hacker, W.: Psychische Regulation von Arbeitstätigkeiten. In: Kleinbeck, U., Schmidt, K.-H. (Hrsg.) Arbeitspsychologie. Hogrefe, Göttingen (2010)

Hacker, W., Richter, P.: Psychische Regulation von Arbeitstätigkeiten - ein Konzept in Entwicklung. In: Frei, F., Udris, I. (Hrsg.) Das Bild der Arbeit. Huber, Bern (1990)

Hackman, J.R., Oldham, G.R.: Development of the job diagnostic survey. J. Appl. Psychol. 60(2), 159-170 (1975)

Karasek, R.: Control in the workplace and its health-related aspects. In: Sauter, S.L., Hurrell, J.J., Cooper, C.L. (Hrsg.) Job Control and Worker Health (129-160). Wiley, Chichester (1989)

Kleinbeck, U., Schmidt, K.-H. (Hrsg.): Arbeitspsychologie. Hogrefe, Göttingen (2010)

Luchman, J.N., González-Morales, M.G.: Demands, control, and support: a meta-analytic review of work characteristics interrelationships. J. Occup. Health Psychol. 18(1), 37-52 (2013)

Lüdtke, A.: Wege aus der Ironie in Richtung ernsthafter Automatisierung. In: Botthof, A., Hartmann, E. A. (Hrsg.) Zukunft der Arbeit in Industrie 4.0. Springer Vieweg, Heidelberg, Berlin (2015)

Misterek, F.: Digitale Souveränität: Technikutopien und Gestaltungsansprüche demokratischer Politik. MPIfG Discussion Paper, No. 17/11. Max Planck Institute for the Study of Societies, Cologne (2017)

Mueller, S.T., Hoffman R.R., Clancey, W., Emrey, A., Klein, G.: Explanation in Human-AI Systems: A Literature Meta-Review, Synopsis of Key Ideas and Publications, and Bibliography for Explainable AI (2019). https://arxiv.org/ftp/arxiv/papers/1902/1902.01876. pdf

Nushi, B., Kamar, E., Horvitz, E.: Towards Accountable AI: Hybrid Human-Machine Analyses for Characterizing System Failure (2018). https://arxiv.org/pdf/1809.07424.pdf

Oesterreich, R.: Handlungsregulation und Kontrolle. Urban \& Schwarzenberg, München (1981) 
Oesterreich, R.: Zur Analyse von Planungs- und Denkprozessen in der industriellen Produktion - Das Arbeitsanalyseinstrument VERA. Diagnostica 30(3), 216-234 (1984)

Osterloh, M.: Handlungsspielräume und Informationsverarbeitung. Huber, Bern (1983)

Posch, R.: Digital sovereignty and IT-security for a prosperous society. In: Werthner, H, van Harmelen, F, (Hrsg.) Informatics in the Future - Proceedings of the 11th European Computer Science Summit (ECSS 2015). Vienna, October 2015. Springer, Berlin (2017)

Rasmussen, J.: Skills, rules, and knowledge; signals, signs, and symbols, and other distinctions in human performance models. IEEE Trans. Syst., Man, and Cyber. 13(3), 257-266 (1983)

Schaar, P.: Globale Überwachung und digitale Souveränität. Z. Außen- Sicherheitspolitik 8(4), 447-459 (2015)

Stubbe, J.: Von digitaler zu soziodigitaler Souveränität. In: Wittpahl, V. (Hrsg.) Digitale Souveränität. Bürger, Unternehmen, Staat. Springer Vieweg Open, Berlin (2017)

SVRV - Sachverständigenrat für Verbraucherfragen: Digitale Souveränität - Gutachten des Sachverständigenrats für Verbraucherfragen. SVRV, Berlin (2017)

Timmers, P.: Ethics of AI and Cybersecurity When Sovereignty is at Stake. Mind. Mach. 29(4), 635-645 (2019). https://doi.org/10.1007/s11023-019-09508-4

Trist, E.L., Bamforth, K.W.: Some social and psychological consequences of the longwall method of coal-getting: an examination of the psychological situation and defences of a work group in relation to the social structure and technological content of the work system. Hum. Relat. 4, 3-38 (1951)

Ulich, E.: Arbeitssysteme als soziotechnische Systeme - Eine Erinnerung. J. Psychologie des Alltagshandelns 6(1), 4-12 (2013)

vbw - Vereinigung der Bayerischen Wirtschaft e. V. (Hrsg.): Digitale Souveränität und Bildung. Gutachten des Aktionsrats Bildung. Waxmann, Münster (2018)

Vicente, K.J., Rasmussen, J.: Ecological interface design: theoretical foundations. IEEE Trans. Syst., Man, and Cyber. 22(4), 589-606 (1992)

Wang. D., Yang, Q., Abdul, A., Lim, B. Y.: Designing theory-driven user-centric explainable AI. CHI 2019, May 4-9, 2019, Glasgow (2019)

Wittpahl, V. (Hrsg.): Digitale Souveränität. Bürger, Unternehmen, Staat. Springer Vieweg Open, Berlin (2017)

Zweig, K.A.: Wo Maschinen irren können - Fehlerquellen und Verantwortlichkeiten in Prozessen algorithmischer Entscheidungsfindung. Bertelsmann Stiftung, Gütersloh (2018)

Zweig, K.A.: Algorithmische Entscheidungen: Transparenz und Kontrolle. Analysen \& Argumente Nr. 338. Konrad-Adenauer-Stiftung, Berlin (2019)

Zweig, K.A., Krafft, T.D.: Fairness und Qualität algorithmischer Entscheidungen. In Mohabbat Kar, R. Thapa, B.E.P, Parycek, P. (Hrsg.) (Un)berechenbar? Algorithmen und Automatisierung in Staat und Gesellschaft. Fraunhofer-Institut für Offene Kommunikationssysteme FOKUS, Kompetenzzentrum Öffentliche IT (ÖFIT), Berlin (2018). https:// nbn-resolving.org/urn:nbn:de:0168-ssoar-57570-1 
Open Access Dieses Kapitel wird unter der Creative Commons Namensnennung 4.0 International Lizenz (http://creativecommons.org/licenses/by/4.0/deed.de) veröffentlicht, welche die Nutzung, Vervielfältigung, Bearbeitung, Verbreitung und Wiedergabe in jeglichem Medium und Format erlaubt, sofern Sie den/die ursprünglichen Autor(en) und die Quelle ordnungsgemäß nennen, einen Link zur Creative Commons Lizenz beifügen und angeben, ob Änderungen vorgenommen wurden.

Die in diesem Kapitel enthaltenen Bilder und sonstiges Drittmaterial unterliegen ebenfalls der genannten Creative Commons Lizenz, sofern sich aus der Abbildungslegende nichts anderes ergibt. Sofern das betreffende Material nicht unter der genannten Creative Commons Lizenz steht und die betreffende Handlung nicht nach gesetzlichen Vorschriften erlaubt ist, ist für die oben aufgeführten Weiterverwendungen des Materials die Einwilligung des jeweiligen Rechteinhabers einzuholen. 